\title{
Type-2 aggregation operators
}

\author{
Zdenko Takáč ${ }^{1}$ \\ ${ }^{1}$ Institute of Information Engineering, Automation and Mathematics \\ Slovak University of Technology in Bratislava, Radlinského 9, 81237 Bratislava, Slovak Republic \\ zdenko.takac@stuba.sk
}

\begin{abstract}
The paper deals with an extension of aggregation operators from the set of real numbers (or interval $[0,1])$ to the set of fuzzy truth values (fuzzy sets in $[0,1])$. We define so-called type-2 aggregation operator and show that an extension of ordinary aggregation operator by convolution is a type- 2 aggregation operator. Finally we show that ordinary aggregation operator, as well as aggregation operator for intervals and for $n$-dimensional intervals are special cases of our type-2 aggregation operator.
\end{abstract}

Keywords: Aggregation operator, Fuzzy truth values, Type-2 fuzzy sets, Type-2 aggregation operator, $n$-Dimensional fuzzy set, Fuzzy multiset

\section{Introduction}

At some point, aggregation plays a fundamental role in all kinds of knowledge based systems [1], [2]. The theory of aggregation of real numbers is well established (see e.g. [3], [4], [5]) and is applied in fuzzy logic systems based on (type-1) fuzzy sets.

Aggregation operators for real numbers were extended to the aggregation operators for intervals ([6], [7]). These generalized aggregation operators are applicable in systems based on the intervalvalued fuzzy sets [8], Atanassov's intuitionistic fuzzy sets [9] and interval type-2 fuzzy sets [10]. Recently, Shang et al. [11] generalized the concept of intervalvalued fuzzy sets to $n$-dimensional fuzzy sets (also called fuzzy multisets) and Bedregal et al. [12] proposed aggregation operator for $n$-dimensional intervals. Note that the $n$-dimensional intervals are the membership grades of $n$-dimensional fuzzy sets.

Zadeh [13] introduced the concept of type-2 fuzzy sets as an extension of type- 1 fuzzy sets. The membership grades of type-2 fuzzy sets are type- 1 fuzzy sets in $[0,1]$ (we will refer to as fuzzy truth values). The type-2 fuzzy sets are very useful in circumstances where it is difficult to determine an exact membership function for a fuzzy set [14]. This makes them to be an attractive tool in many real problems. However, there is no theory allowing us to aggregate fuzzy truth values. This is one of several obstacles for applicability of the systems based on type-2 fuzzy sets. Our goal is to overcome this lack of knowledge.

This article is a first announcement of our research activity concerning with aggregation of fuzzy truth values. The aim of this paper is to propose an aggregation operator for fuzzy truth values (type-2 aggregation operator), and to provide a theoretical basis for the concept of type-2 aggregation operator. Moreover, we show that usual definition of (ordinary) aggregation operator, the definition of aggregation operator for intervals [6], and also the definition of aggregation operator for $n$-dimensional intervals [12] are special cases of our definition of type-2 aggregation operator.

Finally, we show that an $n$-dimensional fuzzy set can be interpreted as a class of $\alpha$-cuts of some fuzzy truth value. Thus, membership grades of type-2 fuzzy sets can be approximated via $n$-dimensional fuzzy sets. Note that this approach corresponds to $\alpha$-plane representation [15] and zSlice representation [16] of type-2 fuzzy sets.

The paper is organized as follows. Section 2 contains basic definitions and notations that are used in the remaining parts of the paper. Section 3 presents the extension of (ordinary) aggregation operator via convolution. In Section 4, we provide the axiomatic basis for type-2 aggregation operator and show that the proposed extension satisfies stated axioms. In Section 5 we show that ordinary aggregation operator, as well as aggregation operator for intervals and for $n$-dimensional intervals are special cases of our type-2 aggregation operator. The conclusions are discussed in Section 6 .

\section{Preliminaries}

In this section we present some basic concepts and terminology that will be used throughout the paper.

A mapping $f: X \rightarrow[0,1]$ is called a fuzzy set (or type-1 fuzzy set) in a set $X$, the value $f(x)$ is called a membership grade of $x$. A fuzzy set $f$ in $X$ is convex if for all $\lambda \in[0,1]$ holds $f\left(\lambda x_{1}+(1-\lambda) x_{2}\right) \geq \min \left(f\left(x_{1}\right), f\left(x_{2}\right)\right)$ where $x_{1}$, $x_{2}$ are arbitrary elements of $X$. A fuzzy set $f$ in $X$ is normal if there exists $x \in X$ such that $f(x)=1$. A crisp set $\operatorname{Ker}(f)=\{x \in X \mid f(x)=1\}$ is called a kernel of $f$. A crisp set $f_{\alpha}=\{x \in X \mid f(x) \geq \alpha\}$, where $\alpha \in] 0,1]$ is called an $\alpha$-cut of $f$.

Definition 1 A function $A:[0,1]^{n} \rightarrow[0,1]$ is called a type-1 aggregation operator on $[0,1]$ if and only if it satisfies the conditions:

(A1) $A(0, \ldots, 0)=0$;

(A2) $A(1, \ldots, 1)=1$; 
(A3) $x_{1} \leq y_{1}, \ldots, x_{n} \leq y_{n}$ implies $A\left(x_{1}, \ldots, x_{n}\right) \leq$ $A\left(y_{1}, \ldots, y_{n}\right)$.

for all $x_{1}, y_{1}, \ldots x_{n}, y_{n} \in[0,1]$.

A type-2 fuzzy set in $X$ is a fuzzy set whose membership grades are type-1 fuzzy sets in $[0,1]$. Let $\mathcal{F}$ denotes a class of all type- 1 fuzzy sets in $[0,1]$. Then type-2 fuzzy set in $X$ is a mapping $\widetilde{f}: X \rightarrow \mathcal{F}$ and elements of $\mathcal{F}$ are called fuzzy truth values. We denote by $\mathcal{F}_{N} / \mathcal{F}_{C} / \mathcal{F}_{N C}$ a class of all normal / convex / normal convex fuzzy truth values, respectively. The algebra of fuzzy truth values $\mathbf{F}=(\mathcal{F}, \sqcup, \sqcap, \mathbf{0}, \mathbf{1}, \sqsubseteq, \preceq)$ is closely describe in $[17]$ and [18], whereas

$$
\begin{aligned}
& (f \sqcup g)(z)=\sup _{x \vee y=z}(f(x) \wedge g(y)), \\
& (f \sqcap g)(z)=\sup _{x \wedge y=z}(f(x) \wedge g(y)), \\
& f \sqsubseteq g \quad \text { iff } f \sqcap g=f, \\
& f \preceq g \quad \text { iff } f \sqcup g=g, \\
& \mathbf{0}(x)= \begin{cases}1 & , \text { if } x=0, \\
0 & , \text { otherwise, }\end{cases} \\
& \mathbf{1}(x)= \begin{cases}1 & , \text { if } x=1, \\
0 & , \text { otherwise. }\end{cases}
\end{aligned}
$$

Let $f \in \mathcal{F}$. Then unary operations

$$
f^{L}(x)=\sup _{y \leq x} f(y) \quad \text { and } \quad f^{R}(x)=\sup _{y \geq x} f(y) .
$$

enable us to express the operations $\sqcup, \sqcap$ and relations $\sqsubseteq, \preceq$ pointwise (see [19], [20], [18]):

$$
\begin{gathered}
f \sqcup g=\left(f \wedge g^{L}\right) \vee\left(f^{L} \wedge g\right)=(f \vee g) \wedge\left(f^{L} \wedge g^{L}\right), \\
f \sqcap g=\left(f \wedge g^{R}\right) \vee\left(f^{R} \wedge g\right)=(f \vee g) \wedge\left(f^{R} \wedge g^{R}\right), \\
f \sqsubseteq g \quad \text { iff } \quad f^{R} \wedge g \leq f \leq g^{R}, \\
f \preceq g \quad \text { iff } \quad f \wedge g^{L} \leq g \leq f^{L} .
\end{gathered}
$$

Moreover, a fuzzy truth value $f$ is convex if and only if $f=f^{L} \wedge f^{R}$ (Proposition 33 in [18]).

\section{Extension of type-1 aggregation operators}

We extend a type-1 aggregation operator. Afterwards we show that under some conditions the extended type-1 aggregation operator preserves normality and convexity of fuzzy truth values.

According to Zadeh's extension principle [13] $n$ ary type-1 aggregation operator $A:[0,1]^{n} \rightarrow[0,1]$ can be extended by the convolution with respect to minimum $\wedge$ and maximum $\vee$ to $n$-ary operator $\widetilde{A}: \mathcal{F}^{n} \rightarrow \mathcal{F}$ as follows:

$$
\begin{aligned}
& \widetilde{A}\left(f_{1}, \ldots, f_{n}\right)(y)= \\
& =\sup _{A\left(x_{1}, \ldots, x_{n}\right)=y}\left(f_{1}\left(x_{1}\right) \wedge \ldots \wedge f_{n}\left(x_{n}\right)\right),
\end{aligned}
$$

where $y, x_{1}, \ldots, x_{n} \in[0,1]$ and $f_{1}, \ldots, f_{n} \in \mathcal{F}$.

Our approach is a generalization of some extensions of t-norms and t-conorms proposed in [18], [21] and [22]. Different approach to the subject used Zhou et al. [23] and proposed so-called type-1 OWA operators (although in our opinion the more fitting name should be 'type-2 OWA operator' and the term 'type-1 OWA operator' should stand for ordinary OWA operator).

Example 1. An extension of arithmetic mean $A\left(x_{1}, \ldots, x_{n}\right)=\sum_{i=1}^{n} x_{i} / n$ is the following aggregation operator for fuzzy truth values:

$\widetilde{A}\left(f_{1}, \ldots, f_{n}\right)(y)=\sup _{\frac{\sum_{i=1}^{n} x_{i}}{n}=y}\left(f_{1}\left(x_{1}\right) \wedge \ldots \wedge f_{n}\left(x_{n}\right)\right)$,

Let $n=2$ and let $f_{1}, f_{2}$ be fuzzy truth values with trapezoidal shapes given by $f_{1}=(0.1,0.3,0.6,0.7)$ and $f_{2}=(0.4,0.5,0.7,0.9)$. Then $\widetilde{A}\left(f_{1}, f_{2}\right)$ is a fuzzy truth value with trapezoidal shape given by $\widetilde{A}\left(f_{1}, f_{2}\right)=(0.25,0.4,0.65,0.8)$. See Figure 1 . We can see that in this case it is sufficient to compute the arithmetic means of four parameters of trapezoids. We will closely discuss when it is possible to consider just a few parameters instead of continuous domain in an upcoming comprehensive article on this subject. There we also give more examples of various extended aggregation operators.

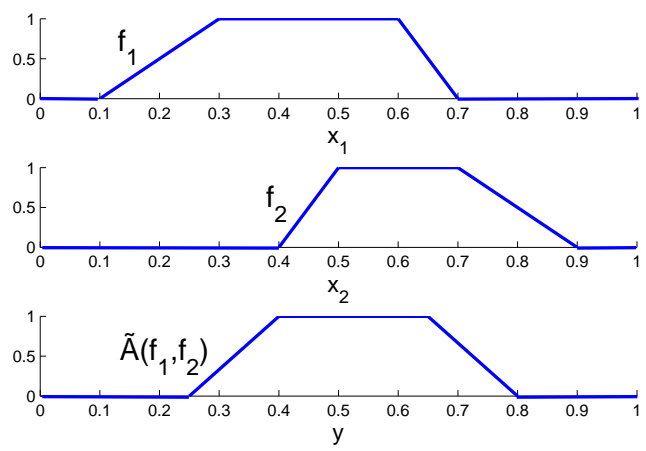

Figure 1: Aggregation of trapezoidal fuzzy truth values $f_{1}, f_{2}$ by extended arithmetic mean $\widetilde{A}\left(f_{1}, f_{2}\right)(y)=$ $\sup _{\frac{x_{1}+x_{2}}{2}=y}\left(f_{1}\left(x_{1}\right) \wedge f_{2}\left(x_{2}\right)\right)$.

For applications of type-2 fuzzy sets, the normal and convex fuzzy truth values are of special significance. So, it is important that normal convex fuzzy truth values $f_{1}, \ldots, f_{n}$ give a normal convex fuzzy truth value $\widetilde{A}\left(f_{1}, \ldots, f_{n}\right)$ whenever $\widetilde{A}$ is an extension of some continuous $n$-ary type- 1 aggregation operator $A$.

Theorem 1 Let $A$ be an n-ary type-1 aggregation operator, let $f_{1}, \ldots, f_{n}$ be normal fuzzy truth values. Then $\widetilde{A}\left(f_{1}, \ldots, f_{n}\right)$ given by (1) is normal fuzzy truth value.

Proof. Let $x_{i}^{p} \in \operatorname{Ker}\left(f_{i}\right)$, for all $i=1, \ldots, n$. Let 


$$
\begin{aligned}
& A\left(x_{1}^{p}, \ldots, x_{n}^{p}\right)=x^{p} . \text { Then } \\
& \qquad \widetilde{A}\left(f_{1}, \ldots, f_{n}\right)\left(x^{p}\right)= \\
& =\sup _{A\left(x_{1}, \ldots, x_{n}\right)=x^{p}}\left(f_{1}\left(x_{1}\right) \wedge \ldots \wedge f_{n}\left(x_{n}\right)\right)= \\
& =f_{1}\left(x_{1}^{p}\right) \wedge \ldots \wedge f_{n}\left(x_{n}^{p}\right)=1 \wedge \ldots \wedge 1=1 .
\end{aligned}
$$

Thus, $x^{p} \in \operatorname{Ker}\left(\widetilde{A}\left(f_{1}, \ldots, f_{n}\right)\right)$, consequently $\widetilde{A}\left(f_{1}, \ldots, f_{n}\right)$ is normal.

q.e.d.

Theorem 2 Let $A$ be a continuous n-ary type-1 aggregation operator, let $f_{1}, \ldots, f_{n}$ be normal convex fuzzy truth values. Then $\widetilde{A}\left(f_{1}, \ldots, f_{n}\right)$ given by (1) is normal convex fuzzy truth value.

Proof. We prove the proposition for $n=2$, the generalization for arbitrary $n$ is straightforward. From Theorem 1 it follows that $\widetilde{A}\left(f_{1}, f_{2}\right)$ is normal. It remains to show that it is also convex, i.e., $\widetilde{A}\left(f_{1}, f_{2}\right)=\left(\widetilde{A}\left(f_{1}, f_{2}\right)\right)^{L} \wedge\left(\widetilde{A}\left(f_{1}, f_{2}\right)\right)^{R}$. The inequality $\leq$ follows from $f \leq f^{L}, f \leq f^{R}$ for all $f \in \mathcal{F}$ (Proposition 3 in [18]), so we are going to show for all $y \in[0,1]$ :

$$
\widetilde{A}\left(f_{1}, f_{2}\right)(y) \geq\left(\left(\widetilde{A}\left(f_{1}, f_{2}\right)\right)^{L} \wedge\left(\widetilde{A}\left(f_{1}, f_{2}\right)\right)^{R}\right)(y) .
$$

From (2) we get:

$$
\begin{aligned}
& \left(\left(\widetilde{A}\left(f_{1}, f_{2}\right)\right)^{L} \wedge\left(\widetilde{A}\left(f_{1}, f_{2}\right)\right)^{R}\right)(y)= \\
& =\left(\sup _{A\left(s_{1}, s_{2}\right)=y}\left(f_{1}\left(s_{1}\right) \wedge f_{2}\left(s_{2}\right)\right)\right)^{L} \wedge \\
& \wedge\left(\sup _{A\left(t_{1}, t_{2}\right)=y}\left(f_{1}\left(t_{1}\right) \wedge f_{2}\left(t_{2}\right)\right)\right)^{R}= \\
& =\left(\sup _{u \leq y} \sup _{A\left(s_{1}, s_{2}\right)=u}\left(f_{1}\left(s_{1}\right) \wedge f_{2}\left(s_{2}\right)\right)\right) \wedge \\
& \wedge\left(\sup _{v \geq y} \sup _{A\left(t_{1}, t_{2}\right)=v}\left(f_{1}\left(t_{1}\right) \wedge f_{2}\left(t_{2}\right)\right)\right)= \\
& =\left(\sup _{A\left(s_{1}, s_{2}\right) \leq y}\left(f_{1}\left(s_{1}\right) \wedge f_{2}\left(s_{2}\right)\right)\right) \wedge \\
& \\
& \wedge\left(\sup _{A\left(t_{1}, t_{2}\right) \geq y}\left(f_{1}\left(t_{1}\right) \wedge f_{2}\left(t_{2}\right)\right)\right),
\end{aligned}
$$

where $s_{1}, s_{2}, t_{1}, t_{2} \in[0,1]$. We denote the very last term by Term. Then (2) can be expressed:

$$
\sup _{A\left(x_{1}, x_{2}\right)=y}\left(f_{1}\left(x_{1}\right) \wedge f_{2}\left(x_{2}\right)\right) \geq \text { Term, }
$$

which we are going to prove. We will consider the following three cases:

1. Let $y \in \operatorname{Ker}\left(\widetilde{A}\left(f_{1}, f_{2}\right)\right)$. This means that $\sup _{\left(x_{1}, x_{2}\right)=y}\left(f_{1}\left(x_{1}\right) \wedge f_{2}\left(x_{2}\right)\right)=1$, hence, (3) holds.
2. Let $y \leq \inf \left(\operatorname{Ker}\left(\widetilde{A}\left(f_{1}, f_{2}\right)\right)\right)$. Now let $A\left(s_{1}, s_{2}\right) \leq y$. Then (see Figure 2):

(i) Let $s_{1} \leq \sup \left(\operatorname{Ker}\left(f_{1}\right)\right), s_{2} \leq \sup \left(\operatorname{Ker}\left(f_{2}\right)\right)$. Then there exists numbers $x_{1}^{0}, x_{2}^{0} \in[0,1]$ such that $A\left(x_{1}^{0}, x_{2}^{0}\right)=y$ and $s_{1} \leq x_{1}^{0} \leq \sup \left(\operatorname{Ker}\left(f_{1}\right)\right)$, $s_{2} \leq x_{2}^{0} \leq \sup \left(\operatorname{Ker}\left(f_{2}\right)\right)$. Recall that $f_{1}, f_{2}$ are convex, so they are increasing on intervals $\left[0, \sup \left(\operatorname{Ker}\left(f_{1}\right)\right)\right], \quad\left[0, \sup \left(\operatorname{Ker}\left(f_{2}\right)\right)\right]$, respectively. Thus, $f_{1}\left(x_{1}^{0}\right) \wedge f_{2}\left(x_{2}^{0}\right) \geq f_{1}\left(s_{1}\right) \wedge f_{2}\left(s_{2}\right)$ and consequently (3) holds.

(ii) Let $s_{1} \leq \sup \left(\operatorname{Ker}\left(f_{1}\right)\right), s_{2}>\sup \left(\operatorname{Ker}\left(f_{2}\right)\right)$. Then there exists $x_{1}^{0}, x_{2}^{0} \in[0,1]$ such that $A\left(x_{1}^{0}, x_{2}^{0}\right)=y$ and $s_{1} \leq x_{1}^{0} \leq \sup \left(\operatorname{Ker}\left(f_{1}\right)\right)$, $s_{2}=x_{2}^{0}$. Recall that $f_{1}$ is increasing on interval $\left[0, \sup \left(\operatorname{Ker}\left(f_{1}\right)\right)\right]$. Thus, $f_{1}\left(x_{1}^{0}\right) \wedge f_{2}\left(x_{2}^{0}\right) \geq$ $f_{1}\left(s_{1}\right) \wedge f_{2}\left(s_{2}\right)$ and consequently (3) holds.

(iii) Let $s_{1}>\sup \left(\operatorname{Ker}\left(f_{1}\right)\right), s_{2} \leq \sup \left(\operatorname{Ker}\left(f_{2}\right)\right)$. Then there exists $x_{1}^{0}, x_{2}^{0} \in[0,1]$ such that $A\left(x_{1}^{0}, x_{2}^{0}\right)=y$ and $s_{1}=x_{1}^{0}, s_{2} \leq x_{2}^{0} \leq$ $\sup \left(\operatorname{Ker}\left(f_{2}\right)\right)$. Recall that $f_{2}$ is increasing on interval $\left[0, \sup \left(\operatorname{Ker}\left(f_{2}\right)\right)\right]$. Thus, $f_{1}\left(x_{1}^{0}\right) \wedge f_{2}\left(x_{2}^{0}\right) \geq$ $f_{1}\left(s_{1}\right) \wedge f_{2}\left(s_{2}\right)$ and consequently (3) holds.

From the previous three cases (i)-(iii) it follows:

$$
\begin{aligned}
& \sup _{A\left(x_{1}, x_{2}\right)=y}\left(f_{1}\left(x_{1}\right) \wedge f_{2}\left(x_{2}\right)\right) \geq \\
& \geq \sup _{A\left(s_{1}, s_{2}\right) \leq y}\left(f_{1}\left(s_{1}\right) \wedge f_{2}\left(s_{2}\right)\right),
\end{aligned}
$$

for all $y \leq \inf \left(\operatorname{Ker}\left(\widetilde{A}\left(f_{1}, f_{2}\right)\right)\right)$.

3. Let $y \geq \sup \left(\operatorname{Ker}\left(\widetilde{A}\left(f_{1}, f_{2}\right)\right)\right)$. The proof of

$$
\begin{aligned}
& \sup _{A\left(x_{1}, x_{2}\right)=y}\left(f_{1}\left(x_{1}\right) \wedge f_{2}\left(x_{2}\right)\right) \geq \\
& \geq \sup _{A\left(t_{1}, t_{2}\right) \geq y}\left(f_{1}\left(t_{1}\right) \wedge f_{2}\left(t_{2}\right)\right),
\end{aligned}
$$

is similar to item 2 with the exception that functions $f_{1}, f_{2}$ are decreasing on intervals [inf $\left.\left(\operatorname{Ker}\left(f_{1}\right)\right), 1\right]$, [inf $\left.\left(\operatorname{Ker}\left(f_{2}\right)\right), 1\right]$, respectively.

Finally, (4) and (5) implies (3).

q.e.d.

\section{Type-2 aggregation operators}

In this section we propose a definition of so-called type-2 aggregation operator, which is a generalization of the standard definition of (type-1) aggregation operator on the set $[0,1]$ to aggregation operator on the set of fuzzy truth values.

Definition 2 Let $(\mathcal{U}, \sqcup, \sqcap, \mathbf{0}, \mathbf{1}, \sqsubseteq, \preceq)$ be a subalgebra of $\mathbf{F}=(\mathcal{F}, \sqcup, \sqcap, \mathbf{0}, \mathbf{1}, \sqsubseteq, \preceq)$. A function $\widetilde{A}$ : $\mathcal{U}^{n} \rightarrow \mathcal{U}$ is called a type-2 aggregation operator on $\mathcal{U}$ if and only if it satisfies the conditions $(\widetilde{A} 1),(\widetilde{A} 2)$ and, for all $f_{1}, \ldots, f_{n}, g_{1}, \ldots, g_{n} \in \mathcal{U}$, at least one of the conditions $(\widetilde{A} 3),\left(\widetilde{A} 3^{\prime}\right)$ :

$(\widetilde{A} 1) \widetilde{A}(\mathbf{0}, \ldots, \mathbf{0})=\mathbf{0}$;

$(\widetilde{A} 2) \widetilde{A}(\mathbf{1}, \ldots, \mathbf{1})=\mathbf{1}$;

( $\widetilde{A} 3) f_{1} \sqsubseteq g_{1}, \ldots, f_{n} \sqsubseteq g_{n}$ implies $\widetilde{A}\left(f_{1}, \ldots, f_{n}\right) \sqsubseteq$ $\widetilde{A}\left(g_{1}, \ldots, g_{n}\right)$, 


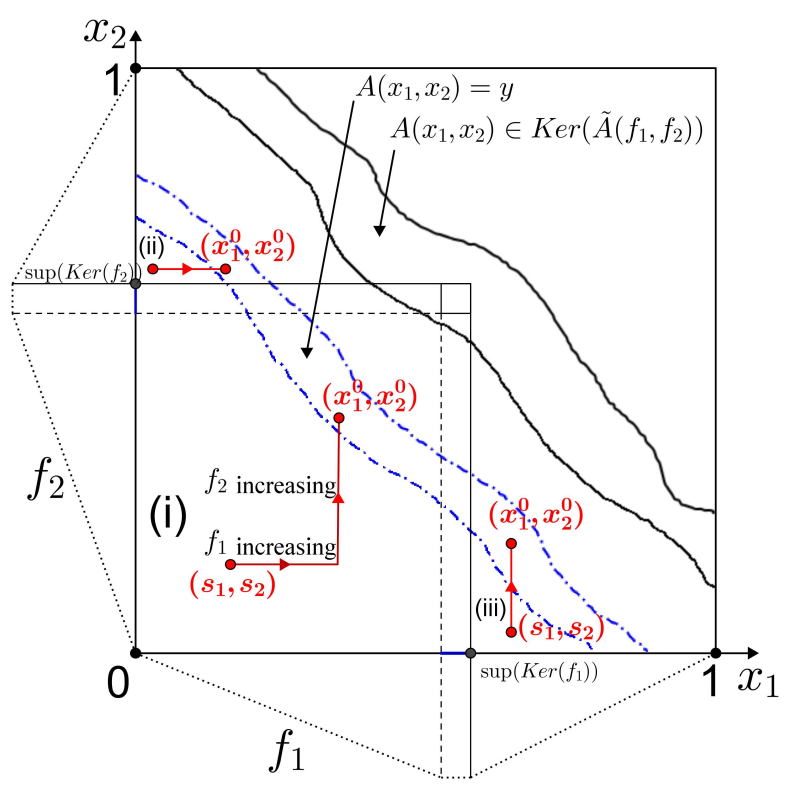

Figure 2: Figure to the proof of Theorem 2.

$\left(\widetilde{A} 3^{\prime}\right) f_{1} \preceq g_{1}, \ldots, f_{n} \preceq g_{n}$ implies $\widetilde{A}\left(f_{1}, \ldots, f_{n}\right) \preceq$ $\widetilde{A}\left(g_{1}, \ldots, g_{n}\right)$.

Recall that for normal convex fuzzy truth values the two orderings coincide, i.e. $\sqsubseteq=\preceq$ (Proposition 19 and Proposition 37 in [18]).

The main result of this section shows that, on the set of normal convex fuzzy truth values, the extended $n$-ary continuous aggregation operator given by (1) is a type-2 aggregation operator.

Theorem 3 Let $A$ be a continuous n-ary type-1 aggregation operator, let $\mathcal{F}_{N C}$ be the set of normal convex fuzzy truth values. Then the extended aggregation operator $\widetilde{A}: \mathcal{F}_{N C}^{n} \rightarrow \mathcal{F}_{N C}$ with

$\widetilde{A}\left(f_{1}, \ldots, f_{n}\right)(y)=\sup _{A\left(x_{1}, \ldots, x_{n}\right)=y}\left(f_{1}\left(x_{1}\right) \wedge \ldots \wedge f_{n}\left(x_{n}\right)\right)$,

where $y, x_{1}, \ldots, x_{n} \in[0,1]$ and $f_{1}, \ldots, f_{n} \in \mathcal{F}_{N C}$, is a type-2 aggregation operator.

Proof. Omitted for the reason of space. The proof will be given in an upcoming comprehensive article on this subject.

Example 2. In Example 1 we showed extended arithmetic mean $A\left(x_{1}, \ldots, x_{n}\right)=\sum_{i=1}^{n} x_{i} / n$, for $n=2$, which is a type-2 aggregation operator for each $n=1,2, \ldots$ Arithmetic mean is a continuous aggregation operator, so, it preserves convexity and normality of fuzzy truth values (see Figure 1).

Now we give an example of discontinuous aggregation operator whose extension is not a type- 2 aggregation operator and does not preserve the convexity. Let $A$ be a binary aggregation operator with:

$$
A\left(x_{1}, x_{2}\right)= \begin{cases}0 & , \text { if }\left(x_{1}, x_{2}\right) \in[0,0.5] \times[0,0.5], \\ 0.3 & \left.\left., \text { if }\left(x_{1}, x_{2}\right) \in[0,0.5] \times\right] 0.5,1\right], \\ 0.7 & \text {,if } \left.\left.\left(x_{1}, x_{2}\right) \in\right] 0.5,1\right] \times[0,0.5], \\ 1 & \left.\left.\left.\left., \text { if }\left(x_{1}, x_{2}\right) \in\right] 0.5,1\right] \times\right] 0.5,1\right]\end{cases}
$$

and $f_{1}, f_{2}, g_{1}, g_{2}$ be trapezoidal fuzzy truth values with the following parameters $f_{1}=$ $(0.3,0.4,0.6,0.7), f_{2}=(0.1,0.3,0.4,0.6), g_{1}=$ $(0.35,0.45,0.65,0.75), \quad g_{2}=(0.6,0.7,0.8,0.9)$. Clearly $f_{1} \sqsubseteq g_{1}$ and $f_{2} \sqsubseteq g_{2}$. Values of $\widetilde{A}\left(f_{1}, f_{2}\right)$ and $\widetilde{A}\left(g_{1}, g_{2}\right)$ are in the second and third row of the following table. Values of $\widetilde{A}\left(f_{1}, f_{2}\right) \sqcap \widetilde{A}\left(g_{1}, g_{2}\right)$ are in the fourth row:

\begin{tabular}{c||c|c|c|c}
$y$ & 0 & 0.3 & 0.7 & 1 \\
\hline \hline$\widetilde{A}\left(f_{1}, f_{2}\right)(y)$ & 1 & 0.5 & 1 & 0.5 \\
\hline$\widetilde{A}\left(g_{1}, g_{2}\right)(y)$ & 0 & 1 & 0 & 1 \\
\hline$\left(\widetilde{A}\left(f_{1}, f_{2}\right) \sqcap \widetilde{A}\left(g_{1}, g_{2}\right)\right)(y)$ & 1 & 1 & 1 & 0.5
\end{tabular}

We can see that neither $\widetilde{A}\left(f_{1}, f_{2}\right)$ nor $\widetilde{A}\left(g_{1}, g_{2}\right)$ is convex. Moreover, $\widetilde{A}$ is not type- 2 aggregation operator, because $\left(\widetilde{A}\left(f_{1}, f_{2}\right) \sqcap \widetilde{A}\left(g_{1}, g_{2}\right)\right) \neq \widetilde{A}\left(f_{1}, f_{2}\right)$, i.e. $\widetilde{A}\left(f_{1}, f_{2}\right) \sqsubseteq \widetilde{A}\left(g_{1}, g_{2}\right)$ does not hold.

\section{Type-2 aggregation operators on various subalgebras of $\mathcal{F}$}

In this section we show that the ordinary aggregation operator, as well as the aggregation operator for intervals and for $n$-dimensional intervals are special cases of our type-2 aggregation operator. We discuss the relation between our generalized aggregation operator given by Definition 2 and some known aggregation operators on the sets isomorphic to some subalgebras of $\mathcal{F}$.

\subsection{Fuzzy grades of (type-1) fuzzy sets}

Let $\mathcal{S}$ be a set of all the singletons from $\mathcal{F}$, i.e.,

$$
f \in \mathcal{S} \quad \text { iff } \quad f(x)= \begin{cases}1 & , \text { if } x=a, \\ 0 & , \text { otherwise }\end{cases}
$$

for some $a \in[0,1]$.

Then $\mathbf{S}=(\mathcal{S}, \sqcup, \sqcap, \mathbf{0}, \mathbf{1}, \sqsubseteq)$ is a subalgebra of $\mathbf{F}$ isomorphic with the algebra $([0,1], \vee, \wedge, 0,1, \leq)$ (see $[18]$ ), i.e. isomorphic with the fuzzy grades of type1 fuzzy sets. If $\mathcal{U}=\mathcal{S}$, Definition 2 coincides with the usual notion of $n$-ary aggregation operator (e.g. $[3],[2])$.

\subsection{Fuzzy grades of interval-valued fuzzy sets}

Let $\mathcal{I}$ be a set of all the characteristic functions of closed subintervals of $[0,1]$, i.e.,

$$
f \in \mathcal{I} \quad \text { iff } \quad f(x)= \begin{cases}1 & , \text { if } x \in[a, b], \\ 0 & , \text { otherwise, }\end{cases}
$$

for some $[a, b] \subseteq[0,1]$.

Then $\mathbf{I}=(\mathcal{I}, \sqcup, \sqcap, \mathbf{0}, \mathbf{1}, \sqsubseteq)$ is a subalgebra of $\mathbf{F}$ isomorphic with the algebra 
$\left([0,1]^{2}, \vee, \wedge,[0,0],[1,1], \leq\right) \quad($ see $[18]), \quad$ i.e. $\quad$ isomorphic with the fuzzy grades of interval-valued fuzzy sets under standard maximum $\vee$, minimum $\wedge$ and ordering $\leq$ for intervals. If $\mathcal{U}=\mathcal{I}$, Definition 2 coincides with the definition of $n$-ary aggregation operator for intervals (e.g. [6], [7]).

\subsection{Fuzzy grades of $n$-dimensional fuzzy sets}

Shang et al. [11] introduced an $n$-dimensional fuzzy set $A$ on $Z$ as a mapping $A: Z \rightarrow[0,1]^{n}$, where $A(z)=\left(A_{1}(z), \ldots, A_{n}(z)\right)$ with $A_{1}(z) \leq A_{2}(z) \leq$ $\ldots \leq A_{n}(z)$ is called an $n$-dimensional interval. Recall that type-1 fuzzy sets and interval-valued fuzzy sets are special cases of $n$-dimensional fuzzy sets for $n=1$ and $n=2$, respectively. One of the possible interpretations is: an n-dimensional interval can be seen as a chain of nested intervals of length $k=\frac{n}{2}$ (for even $n$ ) or $k=\frac{n+1}{2}$ (for odd $n$ ) representing different uncertainty levels on the membership degree [12]. Now, let us interpret these nested intervals as $\alpha$-cuts of some fuzzy truth value $f$ (fuzzy grade of type-2 fuzzy set for some $z \in Z$ ) for $\alpha=\frac{1}{k}, \frac{2}{k}, \ldots, 1$ :

$$
\begin{gathered}
f_{\frac{1}{k}}=\left[A_{1}(z), A_{n}(z)\right], f_{\frac{2}{k}}=\left[A_{2}(z), A_{n-1}(z)\right], \ldots \\
\ldots, f_{1}=\left[A_{k}(z), A_{n-k+1}(z)\right]
\end{gathered}
$$

for even $n$, and

$$
\begin{gathered}
f_{\frac{1}{k}}=\left[A_{1}(z), A_{n}(z)\right], f_{\frac{2}{k}}=\left[A_{2}(z), A_{n-1}(z)\right], \ldots \\
\ldots, f_{1}=\left[A_{k}(z), A_{k}(z)\right]
\end{gathered}
$$

for odd $n$. Now, let $\mathcal{V}$ be, for some fixed $n$, a subset of $\mathcal{F}$ given by:

$$
g \in \mathcal{V} \quad \text { iff } \quad g(x)= \begin{cases}\frac{1}{k} & , \text { if } x \in f_{\frac{1}{k}}-f_{\frac{2}{k}}, \\ \frac{2}{k} & , \text { if } x \in f_{\frac{2}{k}}-f_{\frac{3}{k}}, \\ \vdots & \\ \frac{k-1}{k} & , \text { if } x \in f_{\frac{k-1}{k}}-f_{1}, \\ 1 & , \text { if } x \in f_{1},\end{cases}
$$

for some $f \in \mathcal{F}$.

For example of 6-dimensional fuzzy set and corresponding $g \in \mathcal{V}$ see Figure 3. Clearly, $\mathbf{V}=$ $(\mathcal{V}, \sqcup, \sqcap, \mathbf{0}, \mathbf{1}, \sqsubseteq)$ is a subalgebra of $\mathbf{F}$ isomorphic with the algebra $\left([0,1]^{n}, \vee, \wedge,[0, \ldots, 0],[1, \ldots, 1], \leq\right.$ ), i.e. isomorphic with the fuzzy grades of $n$ dimensional fuzzy sets under standard maximum $\vee$, minimum $\wedge$ and ordering $\leq$ for $n$-dimensional intervals. If $\mathcal{U}=\mathcal{V}$, Definition 2 coincides with the definition of $n$-ary aggregation operator for $n$ dimensional intervals (Definition 3.1 in [12]).

Thus, membership grades of type-2 fuzzy sets can be approximated via $n$-dimensional fuzzy sets, i.e. as classes of $\alpha$-cuts of fuzzy truth values. Consequently, various applications of $n$-dimensional fuzzy sets can be adapted as an approximate applications for type-2 fuzzy sets. For instance, the example
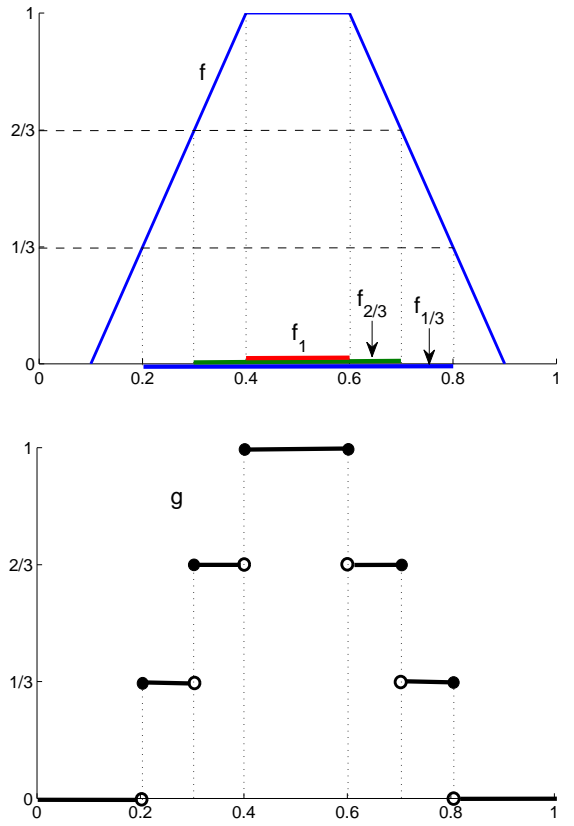

Figure 3: A trapezoidal fuzzy truth value $f$ with parameters $(0.1,0.4,0.6,0.9)$. Its $\alpha$-cuts $f_{\frac{1}{3}}=[0.2,0.8], \quad f_{\frac{2}{3}}=[0.3,0.7]$ and $f_{1}=$ $[0.4,0.6]$ produce 6 -dimensional fuzzy set $A(z)=$ $(0.2,0.3,0.4,0.6,0.7,0.8)$. Corresponding fuzzy truth value $g \in \mathcal{V}$.

of fuzzy multicriteria decision making based on $n$ dimensional fuzzy sets in Section 5 of [12] can be used as an approximate procedure for multicriteria decision making based on type-2 fuzzy sets.

Note that this approach corresponds to $\alpha$-plane representation [15] and zSlice representation [16] of type-2 fuzzy sets.

\section{Conclusions}

We proposed a new concept of type- 2 aggregation operator. These operators aggregate fuzzy truth values, so it is a step to developing fuzzy logic systems based on type-2 fuzzy sets. Type-2 aggregation operator is an extension of known aggregation operators for real numbers, intervals and $n$ dimensional intervals. We also showed that an $n$ dimensional fuzzy set can be interpreted as a class of $\alpha$-cuts of fuzzy truth values. Thus, the membership grades of type-2 fuzzy sets can be approximated via $n$-dimensional fuzzy sets. Consequently, various applications of $n$-dimensional fuzzy sets can be adapted as an approximate applications for type2 fuzzy sets.

This paper is a first announcement of our research activity concerning with aggregation of fuzzy truth values. Our emphasis was on the theoretical side. In an upcoming comprehensive article on this subject we will develop the theoretical aspects in more details, we will also give more examples of various extended aggregation operators, and mainly we will 
study a techniques for computation of type- 2 aggregation operators for some specific kinds of fuzzy truth values, e.g. triangular, trapezoidal, gaussian shapes.

\section{Acknowledgment}

This work was supported in part by a Grant APVV0130-12 and a Grant VEGA 1/0419/13.

\section{References}

[1] M. Detyniecki. Mathematical aggregation operators and their application to video querying. Doctoral thesis - research report 2001-002, Laboratoire d'Informatique de Paris 6, 2000.

[2] T. Calvo, A. Kolesárová, M. Komorníková, and R. Mesiar. Aggregation operators: Properties, classes and construction methods. In Aggregation Operators: New Trends and Applications, T. Calvo, G. Mayor, R. Mesiar (Eds.), Physica-Verlag, Heilderberg, New York, pages 1-104, 2002.

[3] R. Mesiar, A. Kolesárová, T. Calvo, and M. Komorníková. A review of aggregation functions. In Fuzzy Sets and Their Extensions: Representation, Aggregation and Models, H. Bustince et al. (Eds.), Springer, Berlin, pages 121-144, 2008.

[4] D. Dubois and H. Prade. A review of fuzzy set aggregation connectives. Information Sciences, 36(1-2):85-121, 1985.

[5] Ronald R. Yager. Aggregation operators and fuzzy systems modeling. Fuzzy Sets and Systems, 67(2):129 - 145, 1994.

[6] Glad Deschrijver and Etienne E. Kerre. Aggregation operation in interval-valued fuzzy and Atanassov's intuitionistic fuzzy set theory. In Fuzzy Sets and Their Extensions: Representation, Aggregation and Models, H. Bustince et al. (Eds.), Springer, Berlin, pages 183-203, 2008.

[7] Z. Takáč. Subsethood measures for intervalvalued fuzzy sets based on the aggregation of interval fuzzy implications. to appear.

[8] L.A. Zadeh. A theory of approximate reasoning. In J.E. Hayes, D. Michie, and L.I. Mikulich, editors, Machine Intelligence, volume 9, New York, 1979.

[9] K.T. Atanassov. Intuitionistic fuzzy sets. Fuzzy Sets and Systems, 20(1):87-96, 1986.

[10] Nilesh N. Karnik and Jerry M. Mendel. Applications of type-2 fuzzy logic systems to forecasting of time-series. Information Sciences, 120(1-4):89-111, 1999.

[11] Y. Shang, X. Yuan, and E.S. Lee. The n-dimensional fuzzy sets and Zadeh fuzzy sets based on the finite valued fuzzy sets. Computers \& Mathematics with Applications, 60(3):442-463, 2010.
[12] B. Bedregal, G. Beliakov, H. Bustince, T. Calvo, R. Mesiar, and D. Paternain. A class of fuzzy multisets with a fixed number of memberships. Information Sciences, 189:1-17, 2012.

[13] L.A. Zadeh. The concept of a linguistic variable and its application to approximate reasoning-I. Information Sciences, 8(3):199-249, 1975.

[14] N.N. Karnik, J.M. Mendel, and Qilian Liang. Type-2 fuzzy logic systems. IEEE Transactions on Fuzzy Systems, 7(6):643 -658, dec 1999.

[15] F. Liu. An efficient centroid type-reduction strategy for general type-2 fuzzy logic system. Information Sciences, 178(9):2224-2236, 2008.

[16] C. Wagner and H. Hagras. zSlices-towards bridging the gap between interval and general type-2 fuzzy logic. In IEEE International Conference on Fuzzy Systems, Hong Kong, pages 489-497, 2008.

[17] M. Mizumoto and K. Tanaka. Some properties of fuzzy sets of type 2. Information and Control, 31(4):312-340, 1976.

[18] Carol L. Walker and Elbert A. Walker. The algebra of fuzzy truth values. Fuzzy Sets and Systems, 149(2):309 - 347, 2005.

[19] D. Dubois and H. Prade. Possibility theory. Plenum Press, New-York, 1988.

[20] Chih-Hui Chiu and Wen-June Wang. A simple computation of MIN and MAX operations for fuzzy numbers. Fuzzy Sets and Systems, 126(2):273 - 276, 2002.

[21] Zsolt Gera and József Dombi. Exact calculations of extended logical operations on fuzzy truth values. Fuzzy Sets and Systems, 159(11):1309 - 1326, 2008.

[22] B.Q. Hu and C.K. Kwong. On type-2 fuzzy sets and their t-norm operations. no published.

[23] Shang-Ming Zhou, Francisco Chiclana, Robert I. John, and Jonathan M. Garibaldi. Type-1 OWA operators for aggregating uncertain information with uncertain weights induced by type-2 linguistic quantifiers. Fuzzy Sets and Systems, 159(24):3281 - 3296, 2008. 